........................................................

AN. MED. INTERNA (Madrid) Vol. 19, N. $^{\circ} 4$, pp. 192-194, 2002

\title{
Enfermedad del legionario con afectación hepática predominante
}

\author{
A. MAGRO MOLINA, V. PLAZA POQUET, V. GINER GALVAÑ \\ Servicio de Medicina Interna. Hospital Clínico de Valencia. Universidad de Valencia
}

LEGIONNAIRES'DISEASE WITH PREDOMINANT LIVER'S INJURY

\begin{abstract}
RESUMEN
Al igual que con el resto de gérmenes atípicos, son escasos los rasgos de presentación sugestivos de neumonía por Legionella Pneumophila, si bien es casi constante la presencia de fiebre con tos seca y frecuentes la diarrea y afectación del sensorio. Analíticamente es común el hallazgo de hiponatremia e hipertransaminasemia moderada. A diferencia de otros gérmenes atípicos, Legionella Pneumophila presenta diseminación hematógena con afectación sistémica grave más propia de gérmenes típicos y elevada mortalidad sin tratamiento adecuado. El diagnóstico etiológico es difícil y suele alcanzarse tardíamente. Lo dificultoso y tardío del diagnóstico etiológico y la potencial gravedad en pacientes predispuestos aconsejan la antibioterapia empírica. Presentamos un caso de neumonía comunitaria grave por Legionella Pneumophila serogrupo 1 con afectación hepática como manifestación principal y buena respuesta a la antibioterapia empírica con claritromicina y rifampicina. Recomendamos el uso empírico de esta antibioterapia en aquellos procesos neumónicos sin diagnóstico microbiológico y evolución tórpida.
\end{abstract}

PALABRAS CLAVE: Neumonía atípica. Legionella Pneumophila Hipertransaminasemia. Claritromicina. Rifampicina.

\begin{abstract}
Like other pneumonias due to atypical agents, pneumonia due to Legionela Pneumophila has no characteristic clinical facts, although fever and non-productive cough are almost constant and diarrhea with changes in mental status are common. Hyponatremia and moderate tran sient hypertransaminasemia are common too. Severe systemic affectation after hematogenous dissemination similar to those described with typical bacterial pneumonias is a prominent difference with other atypi cal agents, with high mortality rates in the absence of appropriate treat ment. Etiological diagnosis is very difficult and it is normally achieved late in the course of the infection. Because of diagnostic difficulties and potential mortality in predisposed patients, empirical antibiotherapy has been extensively recommended. We present a patient affected by critical community-acquired pneumonia due to Legionela Pneumophila sero group 1 with liver alteration as the main manifestation and good response to empirical antibiotherapy with claritromicine and rifampin. We recommended the empirical use of such therapy in those pneumonias without microbiological diagnosis and torpid evolution.
\end{abstract}

KEY WORDS: Atypical Pneumonia. Legionela Pneumophila. Hyper transaminasemia. Clarithromycin. Rifampin.

Magro Molina A, Plaza Poquet V, Giner Galvañ V. Enfermedad del legionario con afectación hepática predominante. An Med Interna (Madrid) 2002; 19: 192-194.

\section{INTRODUCCIÓN}

Legionella pneumophila es responsable de entre 10-20\% de neumonías comunitarias y $10-50 \%$ de las intrahospitalarias (1). Su aparición suele asociarse a factores de riesgo, en general aquellas situaciones que comportan deterioro de la inmunidad celular (2). Tras su inhalación se reproduce intracelularmente a nivel de macrófagos dando lugar a diseminación hematógena en cerca del $40 \%$ de casos, detectándose su presencia en diversos órganos, preferentemente hígado, bazo, y riñones (3).

La fiebre es casi constante junto con la tos irritativa y el dolor torácico, siendo igualmente frecuente la presencia de diarrea y disminución del nivel de conciencia (4). No obstante, excepto por la marcada afectación sistémica, el cuadro clínico es indistinguible del provocado por otros gérmenes atípi$\cos (5)$. Se han descrito como rasgos bioquímicos asociados la hiponatremia, hipoxemia y elevación de urea, LDH y transaminasas en valores estas últimas nunca superiores a cinco veces los valores de la normalidad. Radiológicamente provoca infiltrados alveolares subpleurales de predominio basal que en ocasiones obligan a descartar el tromboembolismo pulmonar (6).

El diagnóstico suele realizarse ante la positividad de serologías habitualmente solicitadas de forma sistemática, lo que implica un diagnóstico tardío, dado que la seroconversión

Trabajo aceptado: 31 de Octubre de 2000

Correspondencia: Vicente Giner Galvañ. Servicio de Medicina Interna. Hospital Clínico. Avda Blasco Ibáñez, 17. 46010 Valencia. e-mail: giner_vicgal@gva.es. 



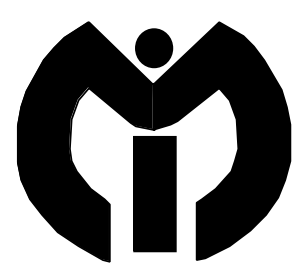

\section{CONGRESO NACIONAL DE MEDICINA INTERNA}

\section{MEDINTERN 2002}

TALLER IbEROAMERICANO "EL INTERNISTA EN LA FormaCión de Profesionales de la Salud”

\section{SEMINARIo INTERNACIONAL SOBRE LA ATENCión INTEGRal de las ENFERMEdades CrónICAS no Transmisibles}

13 al 15 de noviembre de 2002

Palacio de Convenciones de La Habana, Cuba

\section{CUOTA DE INSCRIPCIÓN}

Delegados:

Profesionales:

Estudiantes de Pre-grado:

Acompañantes

$\$ 150,00$ USD

$\$ 100,00$ USD

$\$ 70,00$ USD

\section{ALOJAMIENTO}

Se declara como Hotel Sede del evento el Hotel Palco, anexo al Centro de Convenciones, categoría 4 Estrellas plus y condiciones excepcionales para participantes en eventos.

\section{CORRESPONDENCIA}

Para mayor información pueden contactar con:

Dr. Delfín Pérez Caballero

Presidente

Hospital Clínico Quirúrgico "Hermanos Ameijeiras"

Telf.: (537) 877 6100/877 6011

Fax: (537) 735036

e-mail: vdc@hha.sld.cu

medinn@.hha.sld.cu

delfinpc@infomed.sld.cu

Lic. Migdalia Luna Cisneros

Organización Profesional de Congresos

Palacio de Convenciones de La Habana

Telf.: (537) 2086176

Fax: (537) 2028382 / $2087996 / 2083470$

e-mail: migdalia@palco.cu

migdaluna@hotmail.com 
suele darse a las 2-4 semanas del inicio de la infección (6). La dificultad del diagnósico explica que sólo el $3 \%$ de casos no epidémicos sean diagnosticados (7).

La enfermedad del legionario se asocia a elevadas tasas de mortalidad que llegan al $80 \%$ en los casos intrahospitalarios sin antibioterapia adecuada (8). La elevada mortalidad y lo tardío del diagnóstico hacen recomendable el tratamiento empírico con macrólidos durante 2 semanas, valorando la adición de rifampicina hasta completar tres semanas en casos graves o falta de respuesta a la antibioterapia inicial (9).

Presentamos un caso de enfermedad del legionario en el que la citolisis hepática severa será la manifestación principal del cuadro.

\section{CASO APORTADO}

Varón de 51 años de edad con hipotiroidismo, diabetes mellitus tipo 2 y cardiopatía isquémica que consulta por tos seca y dolor pleurítico de una semana de evolución resistente al tratamiento con cefonicid intramuscular. En la exploración física destacaba TA 90/70 sin signos de hipoperfusión, temperatura axilar $37,2^{\circ} \mathrm{C}$, marcada afectación del estado general y tos seca persistente. En la analítica inicial destacaba 12.360 Leucocitos $/ \mathrm{mm}^{3}$ (62,5\% neutrófilos), Hemoglobina $11,3 \mathrm{~g} / \mathrm{dl}, 572.000$ Plaquetas $/ \mathrm{mm}^{3}$, VSG $73 \mathrm{~mm} / \mathrm{h}$, Índice de Quick 62\%, Glucemia 354 mg/dl, GOT 178 UI/l, GPT 124 UI/l, LDH 1998 UI/l, CK 95 UI/l. La radiografía simple de tórax mostraba cardiomegalia sin otras alteraciones. Se inició tratamiento endovenoso con claritromicina $500 \mathrm{mg} / 12$ horas más ceftriaxona $1 \mathrm{~g} / 24 \mathrm{~h}$.

A las doce horas se evidenció (Fig. 1): GOT 1.530 UI/l, GPT 629 UI/l, LDH 7.110, CK 344 UI/l con fracción Mb de 56 UI/l. Se practicaron gammagrafía con pirofosfato, ecografía abdominal con Doppler y TAC abdominal que resultaron normales. La radiografía simple de tórax practicada pocos días tras el ingreso mostró un infiltrado alveolar basal izquierdo subpleural de morfología poligonal (Figs. 1 y 2) con estudio gammagráfico pulmonar normal. También se practicaron estudios serológicos para los virus VHA, VHB, VHC, CMV y VEB que resultaron negativos.

Ante la mala evolución clínica, persistencia de las alteraciones analíticas y empeoramiento radiológico, se sustituyó la ceftriaxona por imipenem $1 \mathrm{~g} / 8 \mathrm{~h}$ ev más tobramicina $100 \mathrm{mg} / 12 \mathrm{~h}$ ev sin mejoría evidente, por lo que finalmente se añadió rifampicina $600 \mathrm{mg} / 24 \mathrm{~h}$ ev. $\mathrm{Al}$ recibir serología para Legionella pneumophila serotipo 1 a títulos 1/1024 se retiraron tanto el imipenem como la tobramicina. El paciente completó tres semanas de antibioterapia con recuperación clínica y analítica completas. A los dos meses del alta se comprobó la resolución de las alteraciones clínicas y analíticas así como una serología con títulos $1 / 256$.

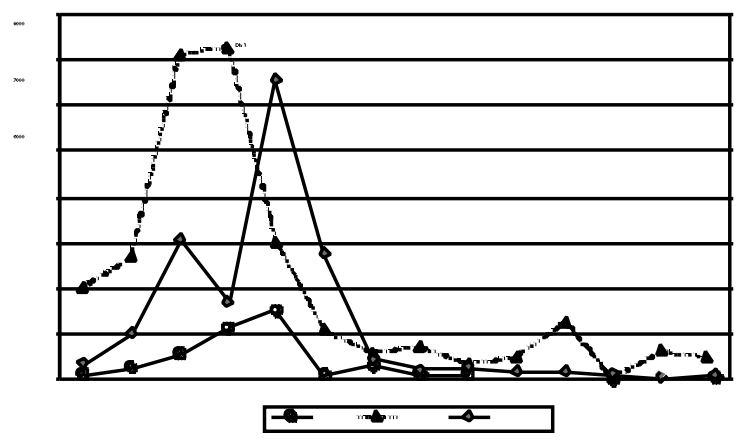

Fig. 1. Evolución analítica. Días referidos respecto al del ingreso (Día 0).

\section{DISCUSIÓN}

El presentado corresponde a un caso aislado de neumonía extrahospitalaria grave por Legionella pneumophila serotipo 1 en un paciente con factores de riesgo (2).

Si bien prominentes en nuestro caso, el dolor torácico y la tos irritativa fueron manifestaciones suficientemente inespecí-
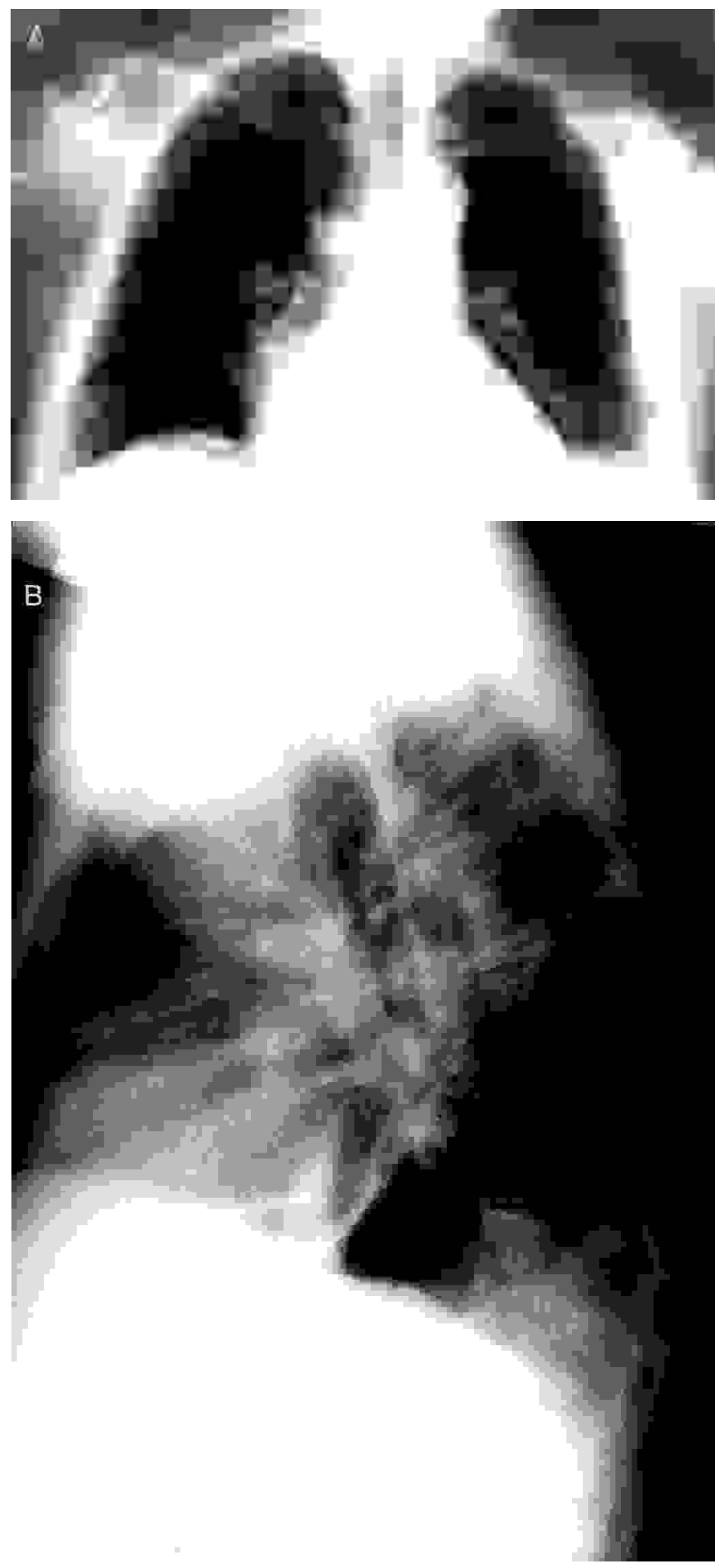

Fig. 2. Neumonía por Legionella pneumophila serotipo 1. Proyecciones frontal (Fig. 2a) y lateral (Fig. 2b) de radiografía simple de tórax mostrando el característico infiltrado subpleural de morfología poligonal. 
ficas para orientar el diagnóstico etiológico, máxime cuando no existió ninguna otra manifestación de las consideradas comúmmente asociadas a Legionella como la fiebre, presente en más del $90 \%$ de casos de enfermedad del legionario (4).

El rasgo más prominente fue la afectación hepática expresada como valores de GOT y GPT diez veces superiores a la normalidad (Fig. 1). La normalidad de la gammagrafía cardíaca, TAC abdominal y Eco-Doppler hepático nos permite descartar causas alternativas del movimiento enzimático como IAM, hígado congestivo, trombosis de venas suprahepáticas o infiltración hepática. La existencia de hipotensión mantenida, presente en cerca del $20 \%$ de casos de neumonía extrahospitalaria por Legionella pneumophila $(4,5)$, plantea la posibilidad de isquemia hepática por bajo gasto; no obstante, en ningún momento el paciente presentó hipoperfusión clínica, y sobre todo, es llamativa la corrección analítica a pesar de la persistencia de la hipotension. Descartadas otras causas y ante el paralelismo entre el perfil evolutivo de los parámetros analíticos y clínicos, nos parece atribuible la hipertransaminasemia a la infección por Legionella pneumophila.

En el $65 \%$ de casos de enfermedad del legionario se detectan elevaciones moderadas de GOT y GPT (10), pero no hemos encontrado ninguna referencia bibliográfica a niveles tan elevados de transaminasas como los observados en nuestro caso. Existen cinco casos descritos en la bibliografía de hepatitis, generalmente anictérica, por Legionella pneumophi la. Suele tratarse de casos graves que afectan a sujetos predispuestos y con evolución rápidamente fatal. El estudio biópsico precoz demuestra marcada colestasis intra y extracelular con escasos focos de necrosis e infiltrados polimorfonucleares neutrófilos y eosinófilos, siendo muy escasos los bacilos intracelulares. Estos hallazgos hacen pensar que la lesión hepática dependería más de endotoxinas que del efecto citopático directo del germen o de la reacción inflamatoria por él inducida (3).

En nuestro caso lo inespecífico de la clínica, y sobre todo, el patrón de alteración analítica hicieron que, si bien la presentación radiológica fuese característica, no se llegase al diagnóstico hasta la recepción de una serología solicitada de forma sistemática ante un proceso neumónico no filiado. La recepción de tal serología sirvió para confirmar la antibioterapia ya empleada empíricamente en un paciente con mala evolución. El caso comentado ilustra la conveniencia de tratamiento antibiótico empírico con macrólidos más rifampicina ante cualquier proceso neumónico no filiado de evolución tórpida, máxime cuando exista alteración enzimática hepática no explicada.

\section{Bibliografía}

1. Blanquer J, Blanquer R, Borrás R, et al. Etiology of community acquired pneumonia in Valencia, Spain: a multicentre prospective study. Thorax 1991; 46: 508-11.

2. Botet ML, Sabrià-Leal M, Sopena N, et al. Role of immunosupression in the evolution of Legionnaires' disease. Clin Infect Dis 1998; 26: 149.

3. Gavilán JC, Bermúdez FJ, Salgado F, Clavijo E. Enfermedad del legionario y hepatitis. Rev Clin Esp 1998; 198: 781.

4. Breiman RF, Butler JC. Legionnaires' disease: clinical, epidemiological, and public health perspectives. Semin Respir Infect 1998; 13: 84-9.

5. Sopena N, Sabrià-Leal M. Comparative study of the clinical presentation of Legionella pneumonia and other community-acquired pneumonias. Chest 1998; 113: 1195-200.
6. Stout JE, Yu VL. Legionellosis. N Engl J Med 1997; 337: 682-7.

7. Marston BJ, Plouffe JF, Breiman RF et al. Preliminary findings of a community-acquired pneumonia incidence study. En: Barbaree JM, Breiman RF, Dufour AP. Legionella, Current Status and Emerging Perspectives. Washington, DC: American Society for Microbiology 1993: 36-7.

8. Heath CH, Grove DI, Looke DFM. Delay in appropiate therapy of Legionella pneumonia associated with increasing mortality. Eur J Clin Microbiol Infect Dis 1996; 15: 286-90

9. Edelstein PH. Antimicrobial chemotherapy for Legionnaries' disease: A review. Clin Infect Dis 1995; 21: 5265-76

10. Pascual JM, Redón J, Borrás R, Vila B, Bertolín V, López Campos V. Neumonía por Legionella pneumophila . Características clínicas de 52 casos. Med Clin 1987; 89: 489-93. 